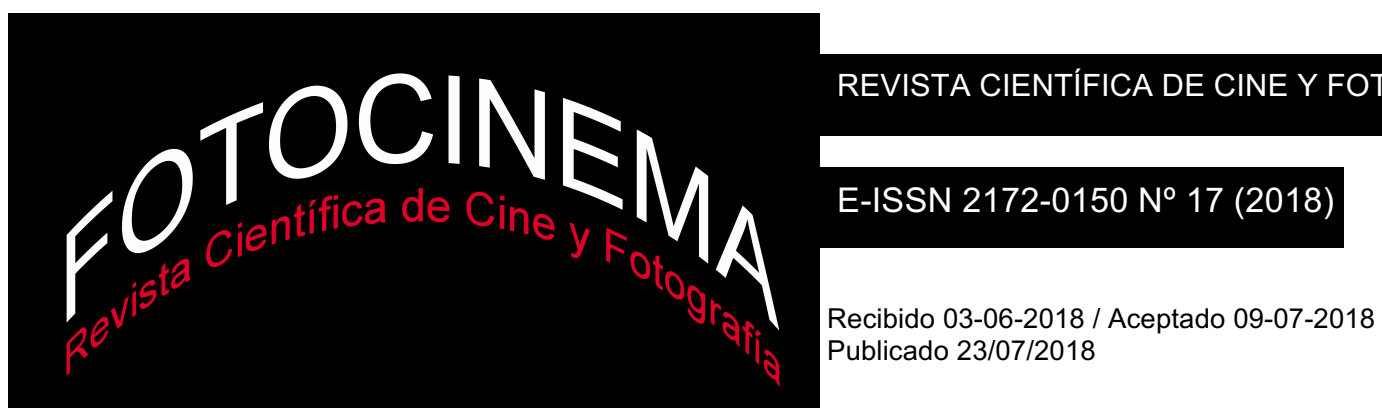

\title{
L'exotisme de la révolution. Michael Christopher Brown (Magnum Photos) et la révolution libyenne, 2011
}

\section{When revolutions are exotic. Michael Christopher Brown (Magnum Photos) and the Lybian revolution, 2011}

\section{El exotismo de la revolución. Michael Christopher Brown (Magnum Photos) y la revolución libiana, 2011}

\begin{abstract}
Jean Kempf
Lyon 2

Résumé:

Jean.Kempf@univ-lyon2.fr

Le photographe américain Michael Christopher Brown s'est rendu en 2011 en Lybie pour y " rencontrer » la révolution en train de s'y dérouler. En 2016, il en tire un livre, Lybian Sugar. La combinaison du choix d'un outil amateur, l'iPhone, et d'une narration qui repose très largement sur l'autobiographie, se veut une tentative de dépassement des limites du photoreportage traditionnel, celui qui a largement informé l'Occident depuis la Guerre d'Espagne. Cet article montre qu'en dépit des qualités intrinsèques de l'ouvrage et d'une certaine virtuosité du photographe, la position éthique qui en ressort reste profondément ambiguë. Elle est plus généralement symptomatique d'une évolution de la photographie occidentale contemporaine qui, malgré des efforts réels, peine à se défaire d'une position auctoriale teintée de post-colonialisme.
\end{abstract}

\begin{abstract}
:
Michael Christopher Brown is a US photographer who went to Lybia in 2011 in order to meet and document the Lybian Revolution. He published his images in a 2016 book, Lybian Sugar. In it, he tried to overcome the limitations of the traditional photo documentary -practiced in Western countries since the Spanish Civil War- by using an amateur camera - the iPhoneand an autobiographical narrative form of his experience there. I argue that the ethical position that emerges from the work remains deeply ambiguous despite the qualities of the book and the photographer's skill. More broadly, I argue that the book is representative of a trend in contemporary Western photography which finds it difficult to go beyond a postcolonial auctorial stance, despite sincere efforts to the contrary.
\end{abstract}

\section{Resumen:}

El fotógrafo estadounidense Michael Christopher Brown fue a Libia en el 2011 a "buscar" la revolución que tenía lugar allí. En 2016, publica Lybian Sugar, el libro de su estancia. El articular una herramienta amateur, el iPhone y una narración que se apoya ampliamente en lo autobiográfico, ambiciona una tentativa de superar los límites del fotoperiodismo tradicional, del que se nutrió Occidente desde la Guerra Civil española. Este artículo revela que, a pesar de las cualidades intrínsecas de la obra y de cierta destreza del fotógrafo, su posición ética no deja de ser profundamente ambigua. En general, es sintomático de una evolución de la fotografía occidental contemporánea que, a pesar de los esfuerzos reales, lucha para deshacerse de una actitud autoral no desprovista de poscolonialismo.

Mots-clefs: Photographie, Etats-Unis, Lybie, printemps arabes, libre photographique, autobiographie, Bildungsroman.

Key words: Photography, United States, Lybia, Arab springs, Photobooks, Autobiography, Bildungsroman.

Palabras clave: fotografía, Estados Unidos, Libia, primaveras árabes, libro de fotografía, autobiografía, Bildungsroman. 
« Les travellings sont affaire de morale »

J.-L. Godard ${ }^{1}$

\section{Prologue}

Si je débute cet article par l'aphorisme mille fois cité de Jean-Luc Godard en réponse à Jacques Rivette, c'est pour inscrire les lignes qui suivent dans la tradition de l'éthique dans l'art². Si l'analyse que l'on va lire est pour une part fondée sur un travail que je mène sur les photographes de conflits, il reprend, dans son esprit et sa méthode, le point de vue de l'essai qui était celui de Rivette ou surtout de Susan Sontag en son temps. Au-delà des critiques dont Sontag a pu faire l'objet, ses livres sur la photographie s'inscrivent dans une position, que je reprends ici à mon compte, qu'aucune pratique artistique, fût-elle la plus formaliste, ne saurait être au-delà de la question éthique, en tout cas ne saurait s'en affranchir. Autrement dit, une révolution dans le viseur ne saurait être une question visuelle, ou plus exactement comme l'affirmait Rivette à propos du film Kapò, la forme visuelle est indissolublement liée à une morale. Ce texte prend donc lui aussi un prétexte pour parler d'autre chose: la révolution libyenne dans le viseur du photographe étatsunien Michael Christopher Brown (né en 1978) comme symptôme de problèmes qui se posent aujourd'hui à la photographie, sa pratique et ses usages.

\section{Michael Christopher Brown en Libye}

En 2011, Michael Christopher Brown se rend en Libye alors qu'éclate, dans la foulée des premiers «printemps arabes », la révolution libyenne, en réalité une guerre civile particulièrement complexe et sanglante qui conduira à une intervention internationale, puis à la chute du dirigeant Mouammar Khadafi, et enfin à la plongée du pays dans un chaos durable et d'une exceptionnelle

\footnotetext{
${ }^{1}$ Jacques Rivette provoqua chez Godard cette réplique-jeu de mots avec sa critique du film de Gillo Pontecorvo, Kapò (1961) et particulièrement de son célèbre travelling sur le visage de la déportée qui se suicide sur les barbelés du camp (de Baecque, 2011).

${ }^{2}$ La question de l'éthique dans l'art (ou pour le formuler dans les termes qui ont cours dans le débat, entre autonomisme et moralisme) a connu dans le monde anglo-américain un renouveau lié à la philosophie analytique et aux écrits de Stanley Cavell (Laugier). Voir Posner, 1997 et Posner 1998 ainsi que Talon-Hugon.
} 
intensité même pour une région qui n'en est pas avare. En 2016, il publie un épais livre de photographies, entrecoupé d'extraits de sa correspondance numérique avec ses proches, qui relate son séjour libyen, Libyan Sugar3. C'est la démarche de Brown telle qu'elle prend forme dans ce livre dont va traiter cet article. Il s'agira de voir quelle iconographie de la révolution ce livre propose, mais surtout comment le récit s'appuie - et même prend prétexte - de cette révolution pour des enjeux tout personnels. Mais ce faisant, on verra aussi comment, dans une large mesure, ce livre fait plus qu'exploiter des archétypes, comment il en est directement issu dans son idéologie profonde et ainsi reproduit un imaginaire occidental dans des formes classiques de la photographie contemporaine.

\section{La révolution exotique : un trope classique en Occident}

Les exemples sont légion de ces jeunes intellectuels que l'idée de révolution non seulement enflamme mais pousse à quitter un environnement souvent confortable - matériellement et mentalement - pour rejoindre, souvent loin du pays natal et familier, une société en éruption. Vue depuis leur base, il leur paraît que va naître ou émerger (les métaphores ne manquent pas) l'Homme Nouveau dans l'insurrection violente - cette donnée est importante. Les citer reviendrait à faire une liste de toutes les révolutions qui, de par le monde, éclosent depuis le début de ce que, justement, on a appelé l'ère des révolutions ${ }^{4}$. Non que la révolte sociale n'existât point avant la fin du XVIIIème siècle, mais ce qui donne à ces nouvelles révoltes leur dimension révolutionnaire est leur lien beaucoup plus que simplement métaphorique avec les mutations « révolutionnaires » dans le domaine des sciences et plus largement de l'économie de ces sociétés (Kuhn, Foucault, Malia)5. Dès lors que ces mouvements se pensent en « révolution » (car la

\footnotetext{
3 Le titre est la reprise d'un graffiti vu Place d'Algérie à Tripoli, qui figure en dernière page du livre (410).

4 Parmi ces convertis et compagnons de route, citons quand même pêle-mêle, Lord Byron rejoignant les Grecs, John Reed rejoignant la Révolution russe, les engagés auprès de la République espagnole, et, durant toute la décolonisation, ceux des colonisateurs luttant contre la colonisation en rejoignant les mouvements d'indépendance. Leur histoire resterait à faire et il est probable qu'elle montrerait une palette de situations et de motivations assez large.

5 Même s'il semble que le terme ait été utilisé dès le XVIIe siècle pour désigner la fameuse «Glorious Revolution » anglaise, le vrai «temps des révolutions » est postérieur. Comme
} 
révolution appelle la théorisation de sa propre pratique sous peine de n'être qu'une simple "révolte»), ils attirent nombre de personnes en apparence étrangères à la cause. Car la plupart du temps et singulièrement dans les cas que nous envisagerons dans cet article, il ne s'agit pas ici de jeunes diasporiques qui rejoignent leurs origines, mais bien d'exotes qui épousent « la cause » (une exogamie en quelque sorte) ou plus précisément qui visitent celle-ci, car c'est bien là le cœur de leur démarche, le retour systématique. Car, pour le converti, le compagnon de route, l'ami de la révolution, le séjour en révolution est rarement définitif car il ne constitue qu'un moment dans sa trajectoire personnelle : quand vient le temps d'une nouvelle introspection, ou d'une réévaluation (en général cruelle) des chemins (dévoyés) qu'emprunte désormais la révolution, le billet retour est là pour assurer à notre exote un retour parmi les siens.

Il est cependant une catégorie bien particulière qui, depuis le début du XXème siècle au moins, se retrouve dans les conflits armés mais aussi sur tous les terrains «à problème» du globe, ce sont les journalistes et, singulièrement dans le cas qui m’intéresse ici, les photographes de presse. Dans une société euro-américaine qui domine le monde, si ce n'est politiquement sous la forme de colonie du moins culturellement à travers le concept «d'humanité » (rien d'humain ne nous étant étranger, nous avons donc besoin d'aller voir et de nous informer sur tout), le journaliste et plus encore le photographe est depuis un bon siècle un vecteur capital de la construction de cette circulation nécessaire pour l'Occident entre intérieur et extérieur, entre « civilisés » et «barbares », ou entre « eux et nous ». Cette démonstration a été mainte fois faite, que cela soit à travers les analyses par exemple des publications des sociétés de géographie, particulièrement le National Geographic, ou des images ethnologiques en général (Gidley, 1992; Lutz, 1995; Walsh 2015).

Avec l'évolution depuis la Seconde Guerre mondiale vers une nouvelle géographie des conflits, le témoin est devenu de plus en plus étranger au terrain. Il n'est sur place que pour un temps limité et revient toujours vers sa base, comme le sociologue ou l'anthropologue avec lequel il partage 
beaucoup : tous les trois sont des formes du personnage moderne (depuis le XIXème siècle) qui incarnent la puissance de l'Occident sur le reste du monde. Depuis la fin de la colonisation, il en est même devenu l'un des derniers représentants, presque le seul à échapper à la destruction massive. Il l'est d'autant plus qu'il ne cherche pas à convertir l'Autre (comme le missionnaire) ou à le soumettre (comme le militaire ou l'administrateur colonial) mais « tout simplement » à le connaître, à le décrire ${ }^{6}$. Il n'y a dans cette description que je livre aucune critique particulière, mais la simple reconnaissance d'une parenté structurelle profonde, presque consubstantielle, entre ces deux formes de voyageurs. Ceci est d'autant plus vrai que les deux se confondent parfois dans le même individu. Et il ne faudrait donc pas voir dans ces trajectoires ainsi décrites la dénonciation d'une «hypocrisie» mais les prolégomènes de l'exégèse / ou de l'interprétation d'une pratique qui définit dans une large mesure à la fois notre conception de l'information et de l'altérité.

\section{Le cas Michael Christopher Brown : le monde pour se trouver}

Michael Christopher Brown a 33 ans quand il arrive en Libye. Ce n'est donc pas un tout jeune homme mais quelqu'un qui a déjà derrière lui quelques années de pratique professionnelle de la photographie. Après des études supérieures, il commence une carrière de photographe fondée, comme celle de dizaines d'autres, sur l'exotisme. Cela va débuter par deux années en Chine (2009-2011), puis, après la Libye le Congo-Kinshasa, la Centrafrique, Cuba. Comme le dit sa biographie "officielle», il travaille alors pour le National Geographic, "completing adventure and cultural stories» (Michael Christopher Brown, Wikipedia). Sa carrière n'est donc en rien différente de la presque totalité des photographes de sa génération dont les critères de «percement » sont aussi aléatoires qu'ils sont flous. Rien ne distingue particulièrement sa trajectoire et surtout ses images. Rien, sauf qu'en 2010, il se met, avant un certain nombre d'autres, à utiliser son téléphone portable (iPhone) pour faire des photographies. Ce deviendra sa

\footnotetext{
${ }^{6}$ Il est vrai cependant que certains de ces exotes manifestent un engagement authentique par des séjours parfois très longs, ou récurrents dans cet ailleurs, établissant parfois leur camp de base sur leur terrain ou aux abords de celui-ci.
} 
marque de fabrique, et pour lui une manière de se distinguer dans un marché qui est grandement saturé en raison de l'offre grandissante et de la demande déclinante.

Mais ce qui rend sa démarche exemplaire (au sens d'exemple-type) est que, bien que s'appuyant sur des sujets « documentaires » (comme la Chine, puis la péninsule de Sakhaline) ou " hot news » (la révolution libyenne), Brown développe déjà une approche éminemment personnelle ou plus exactement psychologisante :

I have always searched for obscure places to escape to and explore. I spent much of my childhood carving trails in mustard and cornfields and wandering the roads and woods of rural Washington State. As I grew, my interest turned to extreme sports and through these activities I reaped fullness in life. Moreover, as a stuttering youth these solo, expressive pursuits were seemingly vital vehicles of communication.

After physical injuries alienated me from this lifestyle and my friends, my father taught me photography. The camera led me inward and I discovered the richness in not only documenting experiences and the physical world but in visually interpreting my surroundings by noticing what was happening inside myself. To see beyond the depressed emotions in my life, photography, paradoxically, showed me a way to recognize the life behind things, a means of expression beyond the physical world7.

Quand vient le temps de la révolution, Brown fait le choix de se rendre en Libye pour des raisons qui, dès le début, sont claires mais pour le moins surprenantes. Il s'agit pour lui de « se trouver ». Très vite il va découvrir que son premier conflit armé, cette révolution qu'il aspire à couvrir en se découvrant, est bien sûr d'une brutalité marquée. Quelques jours plus tard, dans une attaque à la roquette, il est blessé alors qu'à côté de lui sont tués Tim Hetherington et Chris Hondros, deux vétérans de la photographie de conflit. De ce bref séjour dans la révolution, il tire en 2016 Libyan Sugar.

7 http://www.burnmagazine.org/essays/2009/05/michael-christopher-brown-sakhalin-epffinalist/ 


\section{Libyan Sugar, un Bildungsroman dans la révolution}

L'autobiographie, ou plus exactement la subjectivité assumée et revendiquée, a depuis longtemps conquis le documentaire comme marque de sa nouvelle proximité avec l'art et comme réaction paradoxale à l'ère du soupçon qui s'est emparée de la représentation occidentale. Cette tendance a aujourd'hui atteint jusqu'aux photographes de conflit dont on aurait pu penser, au regard de la gravité du sujet, qu’ils en seraient immunisés ${ }^{8}$. Mais si, pour l'essentiel, ceux-ci effectuent un travail qu'ils veulent objectif, dès lors qu'ils sortent du cadre de la stricte « presse » (news) et vont vers le documentaire, nombreux sont ceux qui se rapprochent de l'autobiographie9. Michael Christopher Brown en est un exemple extrême puisque, comme on l'a vu, c'est une sorte de «pacte autobiographique » (dans un sens différent de celui que donne Lejeune à ce terme bien sûr) qui le conduit à partir en Libye ${ }^{10}$.

Par ailleurs, le livre photographique est redevenu ces dernières années un moyen d'expression pour nombre de photographes qui se sentent limités par les contraintes de la publication commerciale, qu'elle soit papier ou en ligne. Le livre offre en effet un espace de présentation des images d'autant plus subtil que le graphisme sur ordinateur offre au concepteur de riches possibilités de simulation et de réalisation. Mais surtout le livre ancre la photographie dans un espace culturel différent en l'associant à la littérature. On peut cependant s'interroger pour savoir qui regarde et a fortiori qui lit ces livres, à part quelques critiques professionnels et amateurs avisés, et quelques photographes ${ }^{11}$ ? Quoi qu'il en soit, Libyan Sugar est un vrai livre,

\footnotetext{
8 On citera par exemple Tim Hetherington et son court-métrage Diary (2010), qui est un rendu personnel et onirique de sa vie de photographe de guerre, film inclus dans l'exposition War/Photography : Images of Armed Conflict and Its Aftermath (Musée des Beaux-Arts de Houston, Texas, 10 novembre 2012-2 février 2013).

9 En publiant, par exemple, des récits autobiographiques comme celui de Lynsey Addario, Tel est mon métier (Addario), ou en se prêtant au jeu de l'interview de leurs collègues.

${ }^{10}$ Son autobiographie, comme toutes les autobiographies, possède ses limites. Un tout petit détail révélateur. Dans le court texte biographique officiel qui figure sur son site et accompagne ses publications, Michael Christopher Brown se présente comme « ayant grandi dans la vallée de la Skagit dans l'État de Washington. » Cela est techniquement vrai mais, formulé ainsi, on l'imagine plutôt fils de paysan, formé par la ruralité. En réalité, Brown est fils de médecin. En privilégiant uniquement l'information géographique sur l'information sociale, on voit bien le type de roman familial qu'il souhaite - consciemment ou non privilégier.

${ }^{11}$ Pour autant, celui-ci reste un objet pour lequel, à ma connaissance, il n'existe pas vraiment d'étude de réception. Car si nombre de livres ont donné lieu à des analyses, je parle ici du
} 
construit comme un récit porté par deux médias - la photo et le texte - et une structure délibérément plus narrative que strictement chronologique (les images ne sont pas organisées exclusivement par dates), c'est-à-dire qu'elle ne répond pas à une pure nécessité externe (la consécution) mais interne (la conséquence).

Mais de quoi parle ce livre ? De Michael Christopher Brown, au prétexte de la révolution: «I made the book for myself, so there was also a need to be transparent, for better or worse, as with a diary. ${ }^{12} »$, ou encore dans la présentation sur son site :

Centered around the 2011 Libyan Revolution, Libyan Sugar is a road trip through a war zone, detailed through photographs, journal entries, and written communication with family and colleagues. A record of Michael Christopher Brown's life both inside and outside Libya during that year, the work is about a young man going to war for the first time and his experience of that age-old desire to get as close as possible to a conflict in order to discover something about war and something about himselfperhaps a certain definition of life and death ${ }^{13}$. (c'est moi qui souligne)

On ne saurait être plus clair : « centré » non pas «sur » (on) mais « à propos, autour de » (around)), ce livre sera bien « une chronique de la vie de Michael Christopher Brown » (a record of Michael Christopher Brown's life). La forme déclarée, «road trip » est ici paradoxale étant donnée la gravité du sujet, car le road trip est avant tout une aventure personnelle (il s'agit de « grandir », de se réaliser (something about himself), d'une forme de rite de passage (young man going to war for the first time), mais aussi un archétype culturel américain, même si ici le texte tente de le raccrocher à un stéréotype anthropologique (age-old desire, a certain definition of life and death). Ce paragraphe est d'ailleurs précédé d'une citation (de la partie narrative du livre) qui relie directement, dans l'évocation thématique et stylistique, le périple libyen de Brown au périple étasunien de Jack Kerouac/Sal Paradise dans On the Road (1957) : "Would Libya be different?

niveau cognitif : comment lisons-nous un livre de photographie? Et non pas qu'y lisonsnous?

${ }_{12} \quad$ https://www.vice.com/en_us/article/5gizi3/libyan-sugar-michael-christopher-brownmagnum-photo

${ }^{13}$ http://michaelchristopherbrown.com/photography/book/ 
Would it be a different world? Something told us so. Something would be there for us." La critique - dithyrambique pour l'essentiel - ne s'y est pas trompée : "Michael Christopher Brown's new book, Libyan Sugar, is about the Libyan revolution. Then again, in many ways, it's not. It's about being a photographer and bearing witness to life-changing events. It's about family and the lengths people go to in order to test themselves. »

Le texte inclus dans le livre, et qui se présente comme un journal mais aussi comme un récit - dont on ne sait pas vraiment donc dans quelle mesure il a été retouché - construit une opposition entre l'avant (2016, p. 11) où le photographe et ses comparses se préparent à des vacances en Libye, et l'après - la fin - tragique ${ }^{14}$. S'il fallait une confirmation supplémentaire, Michael Christopher Brown clarifie d'emblée son propos par une citation sans ambiguïté de Slaughter House Five, un passage particulièrement autobiographique d'un roman qui l'est déjà beaucoup et qui débute par « vous étiez juste des petits enfants pendant la guerre ... pendant la guerre nous étions des puceaux écervelés, juste à la sortie de l'enfance » (Brown, 2016, p. 3) $)^{15}$.

Il explique ensuite sa présence sur le sol libyen par une double généalogie (2016, p. 7) : familiale d'un côté (avec le souvenir de son grand-père engagé pendant la Seconde Guerre mondiale) et nationale/patriotique avec la comparaison entre la lutte des Libyens pour leur liberté et celle de ses « ancêtres révolutionnaires" de 1776 (2016, p. 99) où une vision d'écolier américain vient superposer les deux événements ${ }^{16}$. Le thème de la liberté revient explicitement par deux fois dans le livre, la première sous la forme d'un bref mail de son père (2016, p. 65) et la seconde de la photo de l'épaule d'un Libyen portant un tatouage « freeedom » (2016, p. 333), mais c'est bien la question personnelle qui domine, ce qui nous autorise à le juger non pas sur les modalités de son rapport entre esthétique et vérité (comme pour le journalisme) mais sur le plan de l'éthique (au sens de l'usage fait de la

\footnotetext{
${ }^{14}$ Les chiffres entre parenthèses renvoient aux pages du livre Libyan Sugar.

${ }^{15}$ C'est moi qui traduit Kurt Vonnegut

${ }^{16}$ Il ne rappelle pas en revanche que la jeune République américaine avait conduit deux campagnes militaires, connues sous le nom de Guerres barbaresques, contre ce qui était à l'époque la régence de Tripoli, en 1801-1805 et 1815.
} 
douleur d'autrui) ${ }^{17}$. Même la presse admirative sent bien qu'il faut poser la question de fond. Voici un échange par exemple entre un journaliste et le photographe :

[Vice] You talk in the book about feeling tied to those people you spent time with. A sort of guilt about your ability to leave while the subjects of your work could not. How big a factor was that in keeping you there working?

[MCB] It was a factor, but I stayed in Libya for a number of reasons. I did have compassion for the Libyan situation. There was a feeling that their war was also ours, or mine, because it was-at least at the timeconsidered a fight for dignity and human rights. I identified with these values, and eventually the place and people became a part of me in some way ${ }^{18}$.

Il faut maintenant se demander, au-delà de la révolution prétexte, ce que donne vraiment à voir ce livre ?

\section{Tu n'as rien vu à Misrata}

De la révolution libyenne nous n'apprendrons pas grand-chose. Les instantanés, aussi directs et efficaces soient-ils grâce à la proximité induite par l'iPhone, répètent à l'envi ce que nous étions déjà conditionnés à voir des révolutions du Moyen-Orient. Plus que jamais ici s'applique la phrase dont on ne sait si elle est réellement de Brecht mais qui nous est en tout cas parvenue via Benjamin : voir (et revoir) les usines Krupp ne nous apprend littéralement rien sur les usines Krupp (Benjamin, Brecht). La raison en est profondément anti-aristotélicienne : la forme des choses est indépendante de leur sens, et en s'acharnant à vouloir trouver dans des formes photographiques (l'eidos) la construction possible d'un savoir, le photographe ne peut, bien sûr, que constater que ce qui est mais qui reste en deçà du sens.

${ }_{17}$ La douleur d'autrui n'exploite pas nécessairement l'autre, comme le montrent bien les travaux d'Ariella Azoulay qui complexifient les jugements émis par Sontag dans On Photography, et qu'elle reverra elle-même dans Facing the Pain of Others.

${ }^{18} \mathrm{https} / /$ www.vice.com/en us/article/5gjiji/libyan-sugar-michael-christopher-brownmagnum-photo 
La temporalité pourtant joue un rôle central dans le propos du livre. Elle est à la fois durée et chronologie, et nous est donnée par les messages datés qui ponctuent le texte. Mais elle est en partie bousculée car, si on lit attentivement ces messages, on voit que Brown y fait référence à des scènes qui seront montrées dans des images ultérieures, et les images elles-mêmes ne sont pas placées dans un strict ordre chronologique, marquant ainsi la liberté que se donne le photographe affirmant la primauté de sa propre narration par rapport au «récit vrai» des événements ${ }^{19}$. L'ensemble cependant se présente comme un journal de Libye avec un début, un déroulement d'événements culminant par «l'attaque » (2016, pp. 173-175) et se poursuivant par le retour en Libye après la convalescence, puis une fin qui livre l'explication du titre, ou plutôt sa source, car d'explication il n'en sera guère question (2016, p. 410). Il y aura eu avant un épisode sur la naissance du fils d'un ami auquel le photographe assiste à New York (2016, pp. 246250). Sa valeur intrinsèque est probablement importante pour le photographe et renvoie bien au caractère déjà évoqué profondément « introverti » de l'ouvrage, mais son caractère que l'on qualifiera poliment de convenu et/ou de caricatural en affaiblit l'impact sur le lecteur.

Cela ne doit pas cependant éloigner ce dernier de ce qui viendra ensuite, après le retour cathartique sur les lieux de l'attaque vers une révolution qui, en quelques semaines à peine, a basculé vers un curieux mélange de liesse (2016, pp. 317-333) et de terreurs, avec des pillages et les destructions qui continuent (2016, p. 365). Mais la partie la plus dure de cet après qui n'en n'est pas vraiment un, est celle où l'on voit des malades mentaux dans des hôpitaux en perdition (2016, p. 343sq) (ou bien sont-ce des prisons pour ceux qui ont eu la malchance de rester de l'autre côté (2016, p. 351) ?). On ne saura bien sûr pas si leurs pathologies sont consécutives ou non aux combats ou au régime de Khadafi (on voit en effet dans la série un homme torturé, 2016, pp. 348-49). Ces corps en déshérence participent eux aussi de l'imaginaire de la révolution qui semble toujours se terminer par une immense déception.

${ }^{19}$ Le report en fin d'ouvrage des légendes participe au même processus. 
Le problème pourtant pour le lecteur est que cette temporalité, qui veut signifier la confusion essentielle d'une situation de révolution et/ou de guerre civile, est si longuement étirée que celui-ci n'arrive plus à se reconnecter avec une aventure par trop personnelle et qui dure sans pour autant vraiment l'engager. Cette difficulté à entrer dans la narration a deux causes structurelles: la première est que l'entrée biographique ne tient qu'un moment face au poids du réel; elle a besoin d'être plus consistante pour accrocher le lecteur. De plus, mais ce n'est là qu'une hypothèse, le mode biographique semble plus favorable à la fiction textuelle que visuelle ${ }^{20}$. La seconde est que l'iconographie de la révolution que nous propose Brown, malgré la forme contemporaine de la photographie au téléphone portable, n'est pas radicalement différente de celle à laquelle nous sommes habitués.

Cette iconographie ancienne de la révolution ne s'est pas établie avec la Révolution russe (probablement à cause de sa couverture photographique modeste) mais bien pendant la Guerre d'Espagne, grâce, entre autres mais pas uniquement, à Robert Capa et surtout à la presse illustrée ${ }^{21}$. Les images de Brown reprennent aussi, pour les plus spectaculaires, une esthétique directement issue de la photographie de rue des années 1960 et de livres comme Telex persan de Gilles Peress (1984, pp. 35, 37) ou de certaines images de Raymond Depardon au Liban². On est ici loin des grands photographes du Vietnam ou de la Guerre de Corée pour qui l'image répondait à des critères de sens qui s'exprimaient à travers la force de la composition, et en revanche beaucoup plus près de l'espace fragmenté et hésitant que Robert Frank introduisit avec Les Américains (1958-1959) et que Garry Winogrand et Lee Friedlander portèrent à son paroxysme. Et un peu comme dans leurs images, on ne comprend rien ou si peu : pourquoi la photographie de ce militaire (de carrière) est-elle placée face à celle de jeunes

${ }^{20}$ Il existe cependant quelques essais plus ou moins réussis d'autobiophotographie. On pense à l'œuvre - éminement littéraire - de Denis Roche (1937-2015), ou à celle de Nan Goldin (née en 1953).

${ }^{21}$ La guerre d'Espagne n'est pas la première révolution photographiée mais elle est la première qui fut montrée selon des critères graphiques modernistes, dans la prise de vue et dans sa présentation dans les magazines (Brothers).

${ }^{22}$ Voir les images de Raymond Depardon sur https://pro.magnumphotos.com en particulier "A Christian $\quad$ falangist», $\quad$ Beirut, 1978. http://pro.magnumphotos.com/C.aspx?VP $3=$ SearchResult\&VBID=2K1HZO4DVQAPFO\&S MLS $=1 \& R W=1373 \& R H=759$. 
qui s'essaient au lance-grenade? Que veut dire ce ballet de pieds et de chaussures? Il faudra lire la légende pour lever un peu le voile de cette association mystérieuse.

De même, la correspondance qui scande le déroulé des images est assez superficielle souvent plus de l'ordre du « comment-ça-va-et-vous » que de réels échanges. Parfois arrivent de l'extérieur, via les médias américains et ses parents, des informations sur la situation générale en Libye. Celles-ci sont toutes alarmantes. Face à cette dégradation décrite en termes génériques, Brown oppose des spécificités par ses images qui décrivent alternativement des scènes d'agitation et des morts et des blessés, et des scènes de calme et d'absolue normalité, et qui conduisent à s'interroger sur le sens, sa construction et à terme sur l'information²3. Mais photographier la quotidienneté, à la manière d'un journal, au fil d'images qui croisent notre route, ne produit pas plus de sens. Les images vont, comme l'on dit familièrement, «dans tous les sens. » Alors apparaît la dimension bien connue de l'événement: le témoin de terrain n'en comprend en général ni l'étendue ni la portée. Il est, selon l'expression consacrée, comme Fabrice à Waterloo : « il n'y comprenait rien » (Stendhal, I, 3).

\section{L'être et le nulle part}

Pourtant se rajoute ici dans le récit de Brown une dimension résolument contemporaine que permettent de faire ressentir les conditions même de l'aventure libyenne, à savoir que même dans les pires zones de conflit, les situations les plus extrêmes, nous ne sommes jamais coupés du reste du monde, toujours en lien via des moyens de communication qui nous font être ailleurs que là où nous sommes ${ }^{24}$. La nature même du lieu et de sa signification s'en trouve profondément altérée (on pourrait probablement d'ailleurs parler ici d'une révolution cognitive provoquée par les usages de la

23 Une séquence est particulièrement intéressante. Elle s'ouvre par une image d'un écran de télévision (ABC News) montrant des chasseurs bombardiers en action (114) et se clôt par les corps mutilés et carbonisés de pilotes tombés au sol (120-125). L'inférence visuelle est claire, mais l'information est en réalité fausse : les avions sont ceux de la coalition occidentale, les pilotes abattus étaient des Libyens.

${ }^{24}$ On pense à la scène d'une mort en direct, celle de l'alpiniste néo-zélandais Rob Hall bloqué dans l'Himalaya et conversant jusqu'à la fin avec sa femme par téléphone. Cette aventure est le sujet du film Everest (2015). 
communication numérique sans fil). Mais cette instabilité cognitive a aussi des causes plus matérielles. Ce qui était pour John Reed ou Régis Debray une expatriation et une aventure, est pour nos contemporains une autre expérience ${ }^{25}$. Elle est en effet marquée par des aller-retour entre là-bas et ici, aller-retour réels comme cette sortie en cargo vers Malte (2016, pp. 138-139, 240) ou ensuite vers les Etats-Unis avant son retour sur place (2016, pp246-261) ; mais aller-retour ou plus exactement co-présence des lieux, par le jeu de la technologie : messages instantanés reproduits en captures d'écran (2016, pp. 145-147, 397), et images reçues des Etats-Unis : le père chassant, faisan dans la besace (usage bien différent des armes), le paysage de la vallée de Skagit aux couleurs du Lorrain (2016, pp. 102-103), ou la mère arrosant ses plantes (2016, pp. 156-57) ${ }^{26}$.

La technologie nous donne une impression d'ubiquité, mais nous fait aussi percevoir notre altérité radicale et non, comme on aurait pu le penser, notre communauté fondamentale. Ce message en sourdine court moins dans le livre que dans la lecture que nous en faisons depuis l'Occident, et qu'aucun critique ne semble avoir relevé alors même que Brown en fait un leimotiv (« I’m not a Libyan but an American trying to understand », 2016, p. 99). Cet anti-humanisme contemporain s'inscrit donc en opposition au grand mouvement humaniste qui a habité la photographie de reportage entre la fin de la Seconde Guerre mondiale et la fin des années 1950 et a culminé avec «La grande famille des hommes » (The Family of Man, 1955), mais que l'on trouve chez des photographes par ailleurs très proches du parti communiste, à savoir Henri Cartier-Bresson et son « instant décisif » ou Paul Strand²7.

25 Il est probable que la première vraie occurrence de cette nouvelle « épistémologie » du conflit ait eu lieu avec les guerres de Yougoslavie (1991-2001) et le siège de Sarajevo (19921996).

${ }^{26}$ Ces décalages sont complétés par le choix de naturaliser la révolution d'un côté, et de l'autre de la défamiliariser avec les images de morts en gros plan, ce qui en fait une expérience familière mais incompréhensible. Il en ressort que ce combat que Brown était venu voir n'est vraiment pas le sien.

27 «La grande famille des hommes» (The Family of Man) est une exposition de photographies qui eut lieu au Museum of Modern Art de New York entre janvier et mai 1955, puis qui voyagea en version allégée dans de nombreux pays du monde (Sandeen, Steichen). Cette exposition, très ambitieuse dans sa scénographie, fut un immense succès populaire et un des grands moments de la photographie dite « humaniste ». Le terme "instant décisif » est emprunté par Henri Cartier-Bresson au Cardinal de Retz dans sa préface à son livre Images à la sauvette (1952) comme définition de sa pratique photographique. 


\section{Dieu, que la révolution est jolie !}

Les images les plus crues de cet ouvrage, celles qui ont surtout retenu l'attention de la critique, sont le contrepoint d'un élan visuel profond, sinon joyeux du moins vivant et enthousiaste, que je qualifierais d'une manière de "plastique de la destruction ». Cette plastique comporte à mon sens deux volets. D'un côté une montée vers l'abstraction déjà évoquée supra : même le mortier qui tue devient cratère plus lunaire que mortifère (2016, p. 177). De l'autre une aspiration à un certain surréalisme. Celui-ci s'exprime plutôt dans la première partie de l'ouvrage, mais continue d'émailler le livre tout du long, avec, par exemple cette série d'images de deux jeunes filles jouant à cachecache autour d'un arbre (2016, p. 236), ce masque dans une fête foraine (2016, p. 341) et enfin ce lion du zoo de Tripoli qui fait la couverture de l'ouvrage, impassible, majestueux, hiératique, au regard impénétrable et qui paraît totalement déplacé, comme l'est le zoo lui-même (2016, p. 339) ${ }^{28}$.

Mais si le bonheur n'est guère de la partie, la révolution n'en produit pas moins une excitation visuelle difficile à contrôler. On le sait depuis les images de Andrew Russell des ruines de Richmond (1865), mais aussi de l'Allemagne bombardée (les photos de Walter Hahn de Dresde par exemple) ou du désert libyen, justement, en 1941-1942 de Cecil Beaton²9, la destruction matérielle comporte une dose non négligeable de photogénie. Brown n'échappe donc pas à une forme de sublime des ruines (2016, pp. 207, 373-375) que n'efface pas la souffrance humaine qui est derrière ${ }^{30}$. C'est bien là la contradiction de toute photographie, qui ramène à cette question moins esthétique qu'éthique : que dire de l'image belle d'une chose horrible? Bien entendu cette question n'est pas résolue ici, et on se demande même si elle est abordée, voire reconnue ou même perçue par Brown car même les corps calcinés retrouvés dans les décombres prennent des allures d'œuvre d'art (2016, pp. 214-219). Le sang, dans la première série de blessés, devient peinture abstraite généreusement appliquée sur toute sorte de supports (2016, pp. 192-194, 198-199), indifférenciable de quelque happening des

\footnotetext{
${ }^{28}$ Est-il possible d'y voir, de la part d'un Américain, une référence au lion des studios MGM ? ${ }^{29}$ http://ww2today.com/8th-march-1942-a-different-look-at-the-war-in-the-desert ${ }_{30}$ Même si elles sont suivies par des photos d'identité maculées d'un annuaire d'école (yearbook) et qui fonctionnent comme métaphore de ce qui est arrivé aux corps (380-381).
} 
années 1960. Les portraits de Khadafi généreusement maculés, déchirés, voire les objets lui ayant appartenu (2016, pp. 228-235, 334-337) sont certes le prélude à son lynchage (2016, pp. 313-314), mais apparaissent ici comme autant d'apparitions visuelles dont le lecteur apprécie plus la virtuosité que le caractère brutal et dramatique. Au fond, comme Brown le dit dans son journal : «I felt I was living in a movie, where gestures and dress would have made more sense on a set than on a battlefield » (2016, p. 99).

Si la première partie avait, dans le viseur de Michael Christopher Brown, une dimension surréaliste voire chaotique, la seconde se déploie dans un environnement visuel qui frise très souvent l'abstraction figurative (objets abandonnés, formes, graffitis - 2016, p. 366-367) mais culmine aussi sur les images parmi les plus fortes (en contenu) du livre : les portraits de morts en gros plan comme en écho à la première série de morts (2016, pp. 91, 94, 95) puis la seconde (2016, pp. 118-125); mais c'étaient alors des corps déchiquetés : ici ce sont des visages habités par des yeux morts. Les deux premières séries de cadavres sont évidemment frappantes par leur crudité et le fait qu'elles sont rarement vues dans la presse (d'où ce besoin de transgression, pour le photographe, face à la mort de guerre qu'il découvre). Mais les portraits aux yeux morts le sont bien plus (2016, pp. 305-308), comme l'est cette violence qui s'exerce sur les animaux, manifestation paradoxalement absolue de la folie qui semble s'être emparée de la Libye (un chien étranglé par un fil de fer, p. 371 ; un chameau décapité, p. 299, même si ce dernier a, en fait, été mangé pour célébrer la capture de Khadafi, comme on ne l'apprend qu'en étudiant les légendes en fin de volume.)

Et pourtant, cette folie collective révolutionnaire ne semble pas complètement réelle elle-même. C'est une parade de carnaval, une révolte bricolée, une affaire d'amateurs (2016, p. 128) qui imitent ce qu'ils ont vu dans des films (2016, p. 142), ou d'enfants (2016, pp. 30-49), mais dans un sens plus amusant que tragique de l'enfant-soldat. Les niveaux se mélangent et s'entrechoquent: ce combattant, mitraillette en bandoulière quitte son domicile avec une valise à roulette comme n'importe quel touriste (2016, p. 281), un tel a pris en photo la tête éclatée de son camarade et la montre sur son téléphone Nokia comme on montre des photos de famille (149), et Brown 
s'est fait des selfies après avoir été blessé dans l'attaque (2016, pp. 173-175) comme nous pouvons en faire à tout moment dans notre vie quotidienne ; et ces insurgés combattants d'occasion qui nous apparaissent comme autant de corps en mouvement, presque dansants (2016, pp. 71, 73, 75sq, 87). L'esthétique même de l'iPhone, l’image carrée, de petite taille, une gamme chromatique limitée que compensent des couleurs saturées, une définition suffisante mais faible, et une valorisation du flux et du hasard, donnent à ces images une quotidienneté qui nous les rend accessibles. Au fond, leur absence de spectaculaire nous associe à elles.

\section{En conclusion}

$\mathrm{Au}$ total, ce journal d'une révolution comme on aurait pu l'appeler par antiphrase, constitue la démonstration d'une évolution caractéristique de la photographie contemporaine vers le biographique y compris lorsqu'elle prend comme sujet les grands événements du monde. La différence avec Michael Christopher Brown est que d'ordinaire les photographes reconnaissent tacitement l'incapacité de l'image à dire toute la complexité politique du réel, et qu'ils ne sauraient à travers leurs simples viseurs rendre compte de la réalité, ici la révolution, de ses causes et de ses objectifs. Ils savent qu'ils peuvent tout au plus montrer son état, son déroulement et sa forme temporaire. Mais ils reconnaissent cette incomplétude. Or, malgré les qualités réelles de ce livre que j'ai essayé d'exposer, je dois, pour reprendre le critère de l'éthique annoncé en ouverture, parler de dérive, quoique le terme soit éminemment normatif. Mais c'est bien de cela qu'il s'agit. Avec l'affaiblissement de la notion d'engagement, là où des photographes tels Capa, mais aussi Robert Frank, photographiaient le présent avec une conscience très précise de l'endroit où allaient leurs sympathies, Michael Christopher Brown parti dans une révolution pour se découvrir ne pouvait au fond y trouver que quelques archétypes, ceux-là même que Roland Barthes dénonçait dans «La grande famille des hommes »31. Pire, il produit ici un livre aux multiples affèteries stylistiques, un « bel objet » qui séduit, comme

${ }^{31}$ Que Roland Barthes ait fait une erreur de lecture sur les intentions d'Edward Steichen comme le démontre Eric Sandeen (Sandeen) ne change rien à la force de sa critique si souvent citée (Barthes). 
cela était prévisible, la critique mais dont les qualités intrinsèques en même temps que les limites évidentes en font un compendium de l'état de la photographie aujourd'hui.

\section{Ouvrages cités}

« Michael Christopher Brown », notice Wikipedia.

Addario, L. (2016). Tel est mon métier. Paris : Fayard. (Traduction de This is What I Do.)

Azoulay, A. (2008). The Civil Contract of Photography. New York; Cambridge, MA: Zone Books.

Barthes, R. (1957). « La grande famille des hommes » in Mythologies. Paris : Seuil.

Benjamin, W. (1996 [1931]). Petite histoire de la photographie. Études photographiques 1. http://journals.openedition.org/etudesphotographiques/99, page consultée le 19 avril 2018.

Brecht, B. (1992 [1930]). "Der Dreigroschenprozess. Ein soziologisches Experiment », Werke (éd. W. Hecht). Francfort/Main, Suhrkamp, t. XXI, p. 448-469.

Brothers, C. (1997). War and Photography: A Cultural History. London ; New York : Routledge.

Brown, M. C. (2016). Libyan Sugar. Santa Fe, NM : Twin Palms Publisher. La version feuilletable en ligne se trouve à http://michaelchristopherbrown.com/photography/book/.

Cartier-Bresson, H. (1952). Images à la sauvette. Paris : Verve.

de Baecque, A. (2011). Le cas Kapo: "De l'abjection", ou comment Jacques Rivette forge une morale de la représentation des camps de la mort. Revue d'Histoire de la Shoah, 195,(2), 211-238.

Foucault, M. (1966). Les Mots et les choses. Une archéologie des sciences humaines. Paris : Gallimard.

Gidley, M. (éds.) (1992). Representing Others: White Views of Indigenous Peoples. Exeter: The University of Exeter Press.

Kerouac, J. (1960). Sur la route. Paris : Gallimard. (Traduction de On the Road.)

Kuhn, T. S. (1991). La structure des révolutions scientifiques. (L. Meyer, Trans.) (Nouv. éd. augm. et rev. par l'auteur). Paris: Flammarion.

Laugier, S. (2006). Littérature, philosophie, morale. Fabula-LhT. 1 (février 2006). http://www.fabula.org/lht/1/Laugier.html, page consultée le 19 avril 2018. 
Lutz, C., \& Collins, J. L. (1993). Reading National Geographic. Chicago : University of Chicago Press.

Malia, M. E. (2010). Histoire des Révolutions. Paris : Seuil. (Traduction de History's Locomotives, 2006)

Nussbaum, M. (1998). Exactly and Responsibly: A Defense of Ethical Criticism. Philosophy and Literature, (22).

Peress, G. (1984). Telex persan. Paris : Contrejour. (https://pro.magnumphotos.com/Package/2K7O3RB1IQ48)

Posner, R. (1997). Against Ethical Criticism. Philosophy and Literature, (21).

Posner, R. (1998). Against Ethical Criticism: Part Two. Philosophy and Literature, (22).

Sandeen, E. J. (1995). Picturing an Exhibition: The Family of Man and 1950s America. Albuquerque, NM : University of New Mexico Press.

Sontag, S. (1979). Sur la photographie. Paris : Seuil. (Traduction de On Photography.)

Sontag, S. (2003). Devant la douleur des autres. Paris : C. Bourgois. (Traduction de Facing The Pain of Others.)

Steichen, E. (1955). The Family of Man : the photographic exhibition. New York: Simon \& Schuster. (Nombreuses rééditions de ce catalogue.)

Stendhal (1839). La Chartreuse de Parme, Ière partie, ch. 3.

Talon-Hugon, C. (2009). Morales de l'art. Paris : Presses Universitaires de France.

Vonnegut, K. (1969). Slaughterhouse-five; or, The Children's Crusade, a Duty-dance with Death. New York : Delacorte Press.

Walsh, R. A. (2015). The Geopoetics of Modernism. Gainesville, FL : University Press of Florida. 